\title{
Correction to: Prevalence of musculoskeletal complaints in urban communities in multi-ethnic Suriname: a cross-sectional study with the COPCORD methodology (stage 1, phase 1 and 2)
}

\author{
N. Ho-A-Tham ${ }^{1,2}$ (D) $\cdot$ Y. Vanlandewijck ${ }^{3}$ L. de Donder ${ }^{4} \cdot$ R. Wittoek ${ }^{5} \cdot$ B. Ting-A-Kee ${ }^{6} \cdot$ R. Basantram ${ }^{1} \cdot$ W. Dankaerts $^{2}$
}

Published online: 22 February 2020

(C) International League of Associations for Rheumatology (ILAR) 2020

\section{Correction to: Clinical Rheumatology} https://doi.org/10.1007/s10067-019-04842-5

The footnote of Fig. 2 in the published original version of the above article was missing and the correct figure is presented as follows:

The online version of the original article can be found at https://oi.org/ 10.1007/s10067-019-04842-5

N. Ho-A-Tham

nancyhoatham@gmail.com; nancy.ho-a-tham@uvs.edu

Y. Vanlandewijck

yves.vanlandewijck@kuleuven.be

L. de Donder

liesbeth.de.donder@vub.be

R. Wittoek

Ruth.Wittoek@ugent.be

B. Ting-A-Kee

bvting@yahoo.com

R. Basantram

rashna.basantram@uvs.edu

W. Dankaerts

wim.dankaerts@kuleuven.be
1 Department of Physiotherapy, Faculty of Medical Sciences, Anton de Kom University of Suriname, Paramaribo, Suriname

2 Department of Rehabilitation Sciences, Research Group for Musculoskeletal Rehabilitation, Department of Rehabilitation Sciences, Faculty of Movement and Rehabilitation Sciences, KU Leuven, Leuven, Belgium

3 Department of Rehabilitation Sciences, Research Group of Adapted Physical Activity and Psychomotor Rehabilitation, Department of Rehabilitation Sciences, Faculty of Movement and Rehabilitation Sciences, KU Leuven, Leuven, Belgium

4 Department of Educational Sciences, Faculty of Psychology and Educational Sciences, Vrije Universiteit Brussel, Brussels, Belgium

5 Department of Rheumatology, Ghent University Hospital, Ghent University, Ghent, Belgium

6 Department of Pathology, Faculty of Medical Sciences, Anton de Kom University of Suriname, Paramaribo, Suriname 


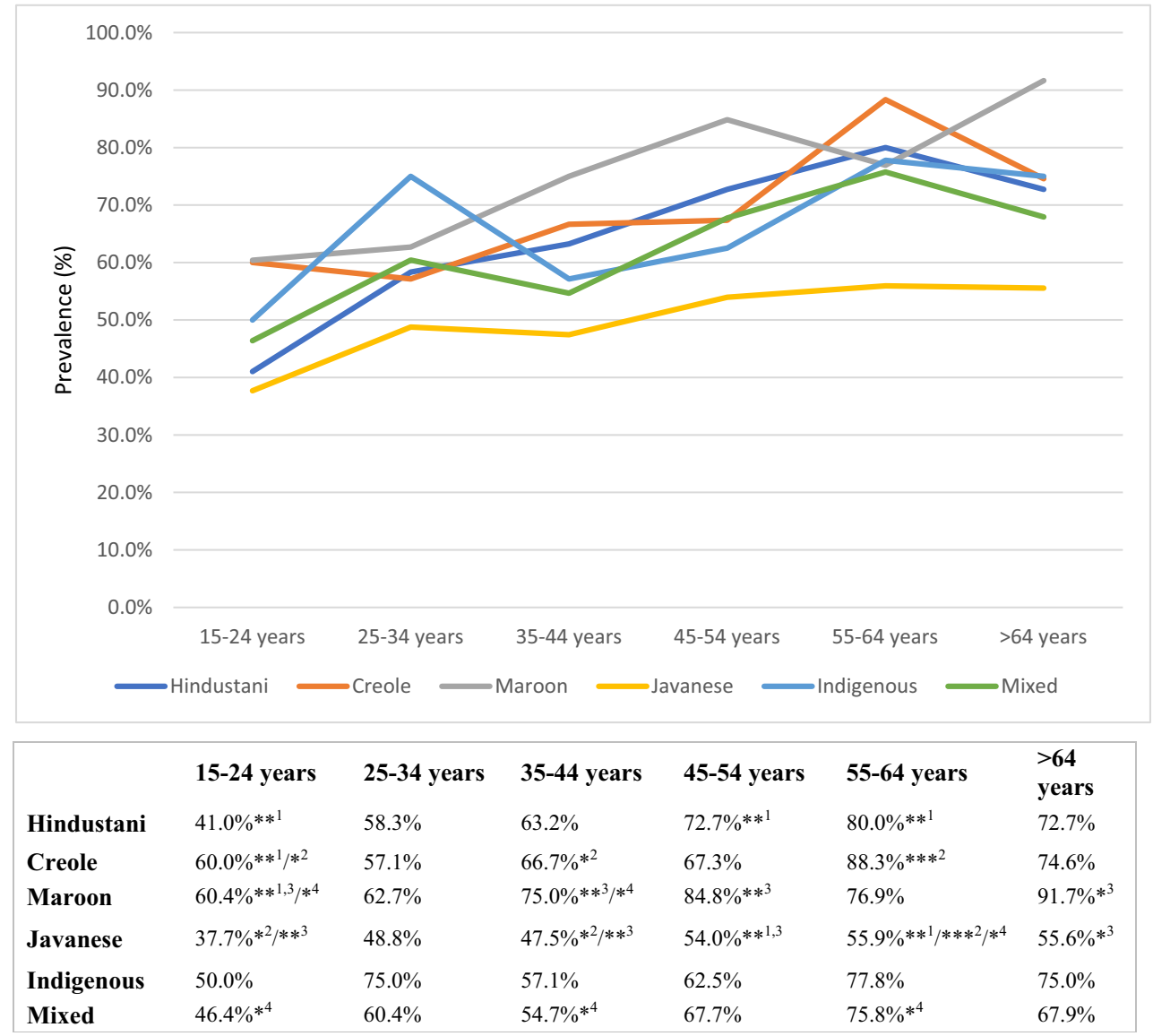

Fig. 2 Prevalence $(\%)$ of musculoskeletal complaints of different ethnic groups by age categories. *** $\mathrm{p} \leq 0.001 * * \mathrm{p} \leq 0.01 * \mathrm{p} \leq 0.05 ; 1-4$ the corresponding numbers next to the significance levels within each age category represent the ethnic groups that differ significantly from each other in prevalence

The original article has been corrected. 